\title{
In-Plane Vibration Response of the Periodic Viaduct on Saturated Soil under Rayleigh Surface Wave
}

\author{
Hai-yan Ju ${ }^{1,2}$ and Ming-fu Fu ${ }^{1,2}$ \\ ${ }^{1}$ School of Civil Engineering and Architecture, Nanchang University, Nanchang, Jiangxi 330031, China \\ ${ }^{2}$ Nanchang Institute of Technology, Nanchang, Jiangxi 330099, China \\ Correspondence should be addressed to Hai-yan Ju; 2005992511@nit.edu.cn
}

Received 10 April 2015; Accepted 1 July 2015

Academic Editor: Xiaobo Qu

Copyright (c) 2015 H.-y. Ju and M.-f. Fu. This is an open access article distributed under the Creative Commons Attribution License, which permits unrestricted use, distribution, and reproduction in any medium, provided the original work is properly cited.

\begin{abstract}
In this study, the in-plane vibration response of the periodic viaduct on saturated soil under Rayleigh surface wave is studied. The Floquet transform method is used to decompose Rayleigh surface wave into a set of spatial harmonic waves. Considering the periodic condition of the viaduct, the wave number domain dynamic response of the periodic viaduct on saturated soil subjected to Rayleigh surface wave excitation is obtained by the transfer matrix method. Then the space domain dynamic response is retrieved by means of the inverse Floquet transform. Numerical results show that when the periodic viaduct is undergoing inplane vibration, there exist three kinds of characteristic waves corresponding to axial compression, transverse shear, and bending vibration. Furthermore, when the frequency of Rayleigh wave is within the pass band of the periodic viaduct, the disturbance propagates over a very long distance and the attenuation of the wave motion far from the source is determined by the characteristic wave with the smallest attenuation, while the vibration attenuates rapidly and propagates in a short distance when the frequency of excitation source is in the range of band gap of periodic structure.
\end{abstract}

\section{Introduction}

As we know, viaduct structure is widely used in engineering. Usually, the viaduct structure generally has equal spans, which means the distance between adjacent piers of a multispan viaduct structure is constant; it can be considered as periodic structure, with the basic element consisting of three parts: a pier, two longitudinal beams, and three linking springs. The period is the distance between two neighboring piers.

The periodic structure has a significant vibration characteristic where energy band exists in periodic structure [1, 2]. When elastic wave propagates in a periodic structure, the vibration within a certain frequency range cannot be passed, which is called band gap, and the vibration within a certain frequency range can be passed, which is called pass band. It provides a new idea for the seismic design and vibration control by using vibration characteristics of periodic structures. With the rational design of the periodic viaduct in geometry and material parameters, it can ensure the frequency of main seismic waves is in the band gap of the viaduct structure, which effectively reduces the structure vibration and damage caused by earthquake wave. Otherwise, if great energy seismic waves are difficult to pass the viaduct structure, sharp increase of energy may be caused in the structural. Therefore, considering the energy band principle of periodic structures, the seismic design and vibration control measures can be achieved by adjusting the structure itself without additional structures.

At present, there are many seismic design methods about viaduct, such as Response Spectrum Method [3-5], TimeHistory Analysis Method [6-8], and Random Vibration Method [9-11]. In the above methods, the viaduct is generally simplified as single degree of freedom system or multidegree of freedom system, and seismic wave is simulated by standing wave. Clearly, the analysis using standing wave method cannot reflect the propagation characteristics of vibration wave in the periodic viaduct. As for the structural vibration induced by seismic wave, the viaduct piers attached to soft foundation were firstly excited. So the seismic energy passed on the viaduct structure varies with the distance from the viaduct pier to vibration source location, usually 
being nonlinear spatial distribution $[12,13]$. Therefore, it is necessary to establish mathematical model which can reflect the propagation characteristics of nonuniform seismic wave in the periodic viaduct so as to provide theoretical basis for seismic design of the viaduct structure.

The periodic viaduct model, consisting of one pier, two longitudinal beams, and three springs, is established to describe the multispan viaduct structure [14, 15]. In this study, as for the Rayleigh surface wave at the bottoms of the piers on the saturated soil, the Floquet transform method is introduced to decompose it into a set of spatial harmonic waves. Considering the periodic condition of the viaduct, the eigenequation for the in-plane vibration of the viaduct in the wave number domain is obtained through transfer matrix method. Then the response in spatial domain of the periodic viaduct on saturated soil under Rayleigh waves can be retrieved by means of the inverse Floquet transform. The influence caused by the characteristic wave propagating in periodic structures and the different Rayleigh waves is discussed.

\section{Control Equations of Periodic Viaduct In-Plane Vibration}

Figure 1 shows the periodic viaduct structure with infinite number of spans. The track, track plates, and beams of each span are simplified as left and right horizontal beams, which are connected by a pier supported on a semi-infinite saturated ground. The intermediate track between neighboring ballasts connecting the left and right horizontal beams is simulated by spring, which is assumed to be able to support axial force, shear force, and bending moment; and the jointing elements between piers and the left or right horizontal beam are also simulated by springs. Therefore, each unit of the periodic viaduct includes a pier, two horizontal beams, and three springs.

Considering the in-plane vibration of the periodic viaduct due to Rayleigh wave, according to Euler-Bernoulli beam theory [16], the motion equations in frequency wave number domain for the pier of the $n$th span element of viaduct can be achieved as follows:

$$
\begin{gathered}
\frac{E_{d} \partial^{2} \widehat{\bar{u}}_{d}^{(n)}(\kappa, z)}{\partial z^{2}}+\rho_{d} \omega^{2} \widehat{\bar{u}}_{d}^{(n)}(\kappa, z)=0, \\
\frac{E_{d} I_{d} \partial^{4} \widehat{\bar{v}}_{d}^{(n)}(\kappa, z)}{\partial z^{4}}-\rho_{d} A_{d} \omega^{2} \widehat{\bar{v}}_{d}^{(n)}(\kappa, z)=0,
\end{gathered}
$$

where $E_{d}, \rho_{d}, A_{d}$, and $I_{d}$ are elastic modulus, density, crosssectional area, and rotational inertia of piers, respectively. $\widehat{\bar{u}}_{d}^{(n)}$ and $\widehat{\bar{v}}_{d}^{(n)}$ are the axial and tangential displacements of piers in frequency wave number domain.

Figure 2 shows the sign conventions for the internal forces of pier internal forces. The cross-sectional axial force $\widehat{\bar{N}}_{d}^{(n)}(\kappa, z)$, shear force $\widehat{\bar{Q}}_{d}^{(n)}(\kappa, z)$, and bending moment

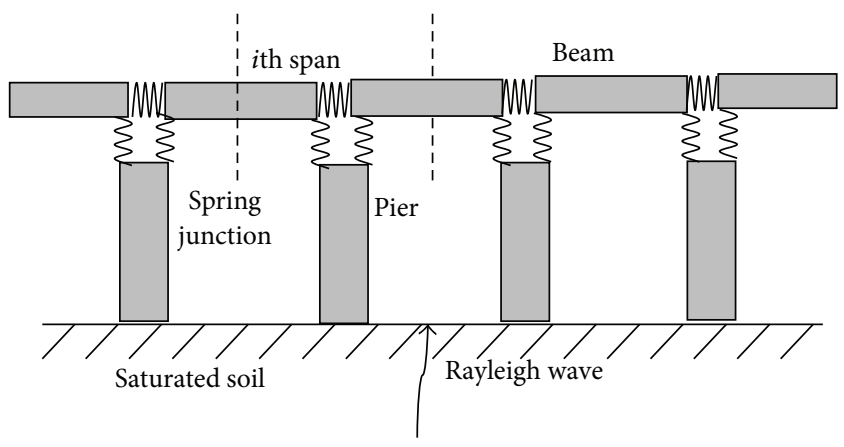

FIGURE 1: A schematic illustration of the periodic viaduct with spring junction subjected to Rayleigh wave.

$\widehat{\bar{M}}_{d}^{(n)}(\kappa, z)$ in frequency wave number domain can be expressed as

$$
\begin{aligned}
& \widehat{\bar{N}}_{d}^{(n)}(\kappa, z)=-\frac{E_{d} A_{d} \partial \widehat{\bar{u}}_{d}^{(n)}}{\partial z}, \\
& \widehat{\bar{M}}_{d}^{(n)}(\kappa, z)=-\frac{E_{d} I_{d} \partial^{2} \widehat{\bar{u}}_{d}^{(n)}}{\partial z^{2}}, \\
& \widehat{\bar{Q}}_{d}^{(n)}(\kappa, z)=-\frac{\partial \widehat{\bar{M}}_{d}^{(n)}}{\partial z} .
\end{aligned}
$$

For the pier of the $n$th span element, the state vector $\widehat{\bar{\psi}}_{d}^{(n)}(\kappa, z)$ at arbitrary position $Z$, which is composed of displacement vector $\widehat{\overline{\mathbf{q}}}_{d}^{(n)}(\kappa, z)$ and internal force vector $\widehat{\overline{\mathbf{f}}}_{d}^{(n)}(\kappa, z)$, is expressed as

$$
\begin{aligned}
& \widehat{\bar{\psi}}_{d}^{(n)}(\kappa, z)=\left\{\widehat{\overline{\mathbf{q}}}_{d}^{(n)}(\kappa, z), \widehat{\overline{\mathbf{f}}}_{d}^{(n)}(\kappa, z)\right\}^{T}, \\
& \widehat{\overline{\mathbf{q}}}_{d}^{(n)}(\kappa, z)=\left\{\widehat{\bar{u}}_{d}^{(n)}(\kappa, z), \widehat{\bar{v}}_{d}^{(n)}(\kappa, z), \widehat{\bar{\theta}}_{d}^{(n)}(\kappa, z)\right\}^{T}, \\
& \widehat{\overline{\mathbf{f}}}_{d}^{(n)}(\kappa, z)=\left\{\widehat{\bar{N}}_{d}^{(n)}(\kappa, z), \widehat{\bar{Q}}_{d}^{(n)}(\kappa, z), \widehat{\bar{M}}_{d}^{(n)}(\kappa, z)\right\}^{T} .
\end{aligned}
$$

Under the condition of in-plane vibration, using EulerBernoulli beam theory and the sign conventions shown in Figure 2(b), the motion equations for horizontal beam of the viaduct in frequency wave number domain are derived as follows:

$$
\begin{gathered}
\frac{E_{b} \partial^{2} \widehat{\bar{u}}_{b}^{(n)}(\kappa, x)}{\partial x^{2}}+\rho_{b} \omega^{2} \widehat{\bar{u}}_{b}^{(n)}(\kappa, x)=0, \\
\frac{E_{b} I_{b} \partial^{4} \widehat{\bar{v}}_{b}^{(n)}(\kappa, x)}{\partial x^{4}}-\rho_{b} A_{b} \omega^{2} \widehat{\bar{v}}_{b}^{(n)}(\kappa, x)=0,
\end{gathered}
$$

where $E_{b}, \rho_{b}, A_{b}$, and $I_{b}$ are elastic modulus, density, crosssectional area, and rotational inertia of horizontal beams, respectively, $\widehat{\bar{u}}_{b}^{(n)}$ and $\widehat{\bar{v}}_{b}^{(n)}$ are axial and tangential displacements of horizontal beams in frequency wave number domain. 


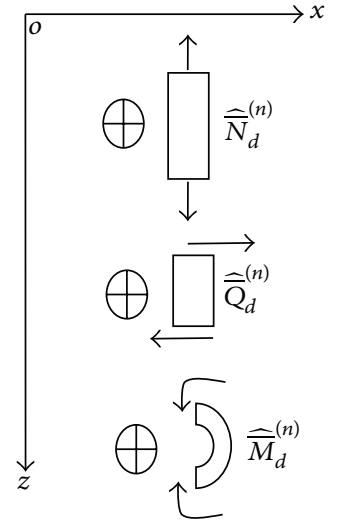

(a)

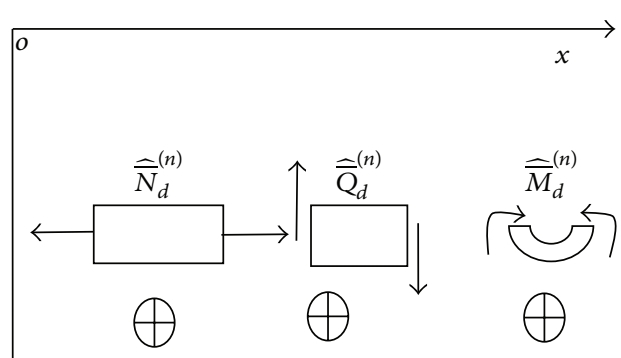

(b)

Figure 2: The sign conventions for the internal forces of a pier and beam. (a) The sign conventions for the internal forces of a pier. (b) The sign conventions for the internal forces of a beam.

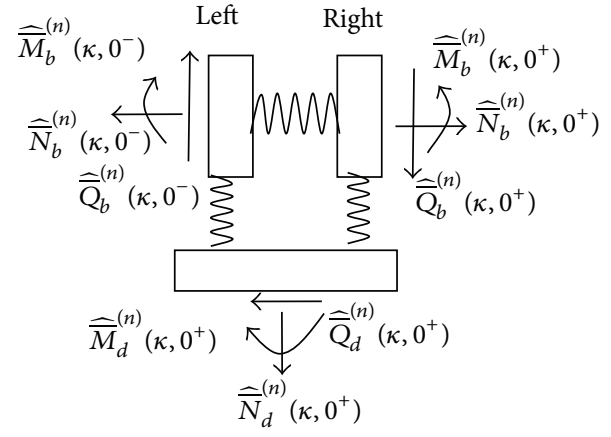

(a)

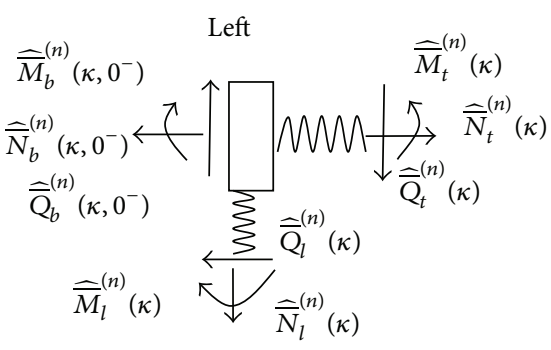

(b)

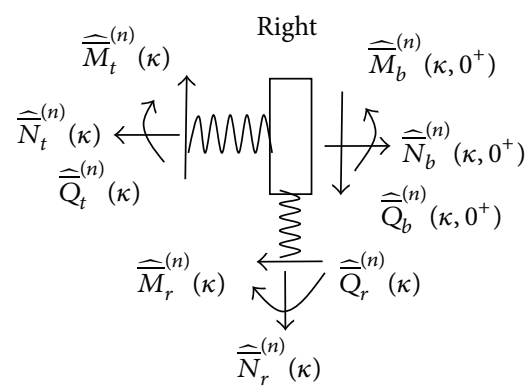

(c)

FIgURE 3: The illustration for the junction linking the pier with the left and right beams undergoing in-plane vibration. (a) The overall beambeam-pier junction. (b) The end of the left beam. (c) The end of the right beam.

According to Euler-Bernoulli beam theory [16], the transfer matrix of the pier and horizontal beam system can be deduced; detailed derivation of (5) can be found in $\mathrm{Lu}$ and Yuan [15]. Consider

$$
\mathbf{T}^{(d)}(z)=\left[\begin{array}{cccccc}
T_{11} & 0 & 0 & T_{14} & 0 & 0 \\
0 & T_{22} & T_{23} & 0 & T_{25} & T_{26} \\
0 & T_{32} & T_{33} & 0 & T_{35} & T_{36} \\
T_{41} & 0 & 0 & T_{44} & 0 & 0 \\
0 & T_{52} & T_{53} & 0 & T_{55} & T_{56} \\
0 & T_{62} & T_{63} & 0 & T_{65} & T_{66}
\end{array}\right]
$$

As for beam-beam junction of the $n$th span in Figure 3, assuming that $k_{t}^{(t)}, k_{t}^{(s)}$, and $k_{t}^{(b)}$ are axial compression stiffness, shear stiffness, and rotational stiffness of beam-beam connection spring, respectively, the axial force $\widehat{\bar{N}}_{t}^{(n)}(\kappa)$, shear force $\widehat{\bar{Q}}_{t}^{(n)}(\kappa)$, and bending moment $\widehat{\bar{M}}_{t}^{(n)}(\kappa)$ of beam-beam connection spring in frequency wave number domain are expressed as

$$
\begin{aligned}
& \widehat{\bar{N}}_{t}^{(n)}(\kappa)=k_{t}^{(t)}\left[\hat{\bar{u}}_{b}^{(n)}\left(\kappa, 0^{+}\right)-\widehat{\overline{\bar{u}}}_{b}^{(n)}\left(\kappa, 0^{-}\right)\right], \\
& \widehat{\bar{Q}}_{t}^{(n)}(\kappa)=k_{t}^{(s)}\left[\widehat{\bar{v}}_{b}^{(n)}\left(\kappa, 0^{+}\right)-\widehat{\overline{\bar{v}}}_{b}^{(n)}\left(\kappa, 0^{-}\right)\right], \\
& \widehat{\bar{M}}_{t}^{(n)}(\kappa)=-k_{t}^{(b)}\left[\hat{\bar{\theta}}_{b}^{(n)}\left(\kappa, 0^{+}\right)-\widehat{\bar{\theta}}_{b}^{(n)}\left(\kappa, 0^{-}\right)\right],
\end{aligned}
$$

where $0^{-}$and $0^{+}$are the left and the right of the connection spring, respectively.

Similarly, for the connection spring linking the pier with the left beam and the right beam, assuming that $k_{l}^{(t)}$, $k_{l}^{(s)}$, and $k_{l}^{(b)}$ are axial compression stiffness, shear stiffness, and rotational stiffness of left beam-pier connection spring, respectively, $k_{r}^{(t)}, k_{r}^{(s)}$, and $k_{r}^{(b)}$ for right beam-pier connection spring, as shown in Figure 3 , the axial force $\left(\widehat{\bar{N}}_{l}^{(n)}\right.$ and $\left.\widehat{\bar{N}}_{r}^{(n)}\right)$, shear force $\left(\widehat{\bar{Q}}_{l}^{(n)}\right.$ and $\left.\widehat{\bar{Q}}_{r}^{(n)}\right)$, and bending 
moment $\left(\widehat{\bar{M}}_{l}^{(n)}\right.$ and $\left.\widehat{\bar{M}}_{r}^{(n)}\right)$ of the connection spring linking the pier with the left and right beams are expressed as

$$
\begin{aligned}
& \widehat{\bar{N}}_{l}^{(n)}(\kappa)=k_{l}^{(t)}\left[\widehat{\bar{u}}_{d}^{(n)}\left(\kappa, 0^{+}\right)-\widehat{\bar{v}}_{b}^{(n)}\left(\kappa, 0^{-}\right)\right], \\
& \widehat{\bar{Q}}_{l}^{(n)}(\kappa)=-k_{l}^{(s)}\left[\widehat{\bar{v}}_{d}^{(n)}\left(\kappa, 0^{+}\right)-\widehat{\bar{u}}_{b}^{(n)}\left(\kappa, 0^{-}\right)\right], \\
& \widehat{\bar{M}}_{l}^{(n)}(\kappa)=-k_{l}^{(b)}\left[\widehat{\bar{\theta}}_{d}^{(n)}\left(\kappa, 0^{+}\right)+\widehat{\bar{\theta}}_{b}^{(n)}\left(\kappa, 0^{-}\right)\right], \\
& \widehat{\bar{N}}_{r}^{(n)}(\kappa)=k_{r}^{(t)}\left[\widehat{\bar{u}}_{d}^{(n)}\left(\kappa, 0^{+}\right)-\widehat{\bar{v}}_{b}^{(n)}\left(\kappa, 0^{+}\right)\right], \\
& \widehat{\bar{Q}}_{r}^{(n)}(\kappa)=-k_{r}^{(s)}\left[\widehat{\bar{v}}_{d}^{(n)}\left(\kappa, 0^{+}\right)-\widehat{\bar{u}}_{b}^{(n)}\left(\kappa, 0^{+}\right)\right], \\
& \widehat{\bar{M}}_{r}^{(n)}(\kappa)=-k_{r}^{(b)}\left[\widehat{\bar{\theta}}_{d}^{(n)}\left(\kappa, 0^{+}\right)+\widehat{\bar{\theta}}_{b}^{(n)}\left(\kappa, 0^{+}\right)\right] .
\end{aligned}
$$

According to Figure 3, without considering the weight of the connection springs, the connection springs linking the pier with the left and right beams meet the following balance equations:

$$
\begin{aligned}
& -\widehat{\bar{N}}_{b}^{(n)}\left(\kappa, 0^{-}\right)+\widehat{\bar{N}}_{b}^{(n)}\left(\kappa, 0^{+}\right)-\widehat{\bar{Q}}_{d}^{(n)}\left(\kappa, 0^{+}\right)=0, \\
& -\widehat{\bar{Q}}_{b}^{(n)}\left(\kappa, 0^{-}\right)+\widehat{\bar{Q}}_{b}^{(n)}\left(\kappa, 0^{+}\right)+\widehat{\bar{N}}_{d}^{(n)}\left(\kappa, 0^{+}\right)=0, \\
& \widehat{\bar{M}}_{b}^{(n)}\left(\kappa, 0^{-}\right)-\widehat{\bar{M}}_{b}^{(n)}\left(\kappa, 0^{+}\right)+\widehat{\bar{M}}_{d}^{(n)}\left(\kappa, 0^{+}\right)=0,
\end{aligned}
$$

where $\widehat{\bar{N}}_{b}^{(n)}, \widehat{\bar{Q}}_{b}^{(n)}$, and $\widehat{\bar{M}}_{b}^{(n)}$ are the internal forces of horizontal beam; $\widehat{\bar{N}}_{d}^{(n)}, \widehat{\bar{Q}}_{d}^{(n)}$, and $\widehat{\bar{M}}_{d}^{(n)}$ are the internal forces of pier.

The connection spring of the left beam-pier and that of the right beam-pier meet the following balance equations:

$$
\begin{aligned}
& \widehat{\bar{N}}_{b}^{(n)}\left(\kappa, 0^{-}\right)=\widehat{\bar{N}}_{t}^{(n)}(\kappa)-\widehat{\bar{Q}}_{l}^{(n)}(\kappa), \\
& \widehat{\bar{Q}}_{b}^{(n)}\left(\kappa, 0^{-}\right)=\widehat{\bar{Q}}_{t}^{(n)}(\kappa)+\widehat{\bar{N}}_{l}^{(n)}(\kappa), \\
& \widehat{\bar{M}}_{b}^{(n)}\left(\kappa, 0^{-}\right)=\widehat{\bar{M}}_{t}^{(n)}(\kappa)-\widehat{\bar{M}}_{l}^{(n)}(\kappa), \\
& \widehat{\bar{N}}_{b}^{(n)}\left(\kappa, 0^{+}\right)=\widehat{\bar{N}}_{t}^{(n)}(\kappa)+\widehat{\bar{Q}}_{r}^{(n)}(\kappa), \\
& \widehat{\bar{Q}}_{b}^{(n)}\left(\kappa, 0^{+}\right)=\widehat{\bar{Q}}_{t}^{(n)}(\kappa)-\widehat{\bar{N}}_{r}^{(n)}(\kappa), \\
& \widehat{\bar{M}}_{b}^{(n)}\left(\kappa, 0^{+}\right)=\widehat{\bar{M}}_{t}^{(n)}(\kappa)+\widehat{\bar{M}}_{r}^{(n)}(\kappa) .
\end{aligned}
$$

\section{Wave Field Solution of Saturated Soil under Rayleigh Surface Wave}

3.1. Biot's Theory and Helmhotlz Vector Decomposition. According to the theory of saturated soil $[12,13]$, the constitutive equations of porous media have the following:

$$
\begin{aligned}
\sigma_{i j} & =2 \mu \varepsilon_{i j}+\lambda \delta_{i j} e-\alpha \delta_{i j} p, \\
p_{f} & =-\alpha M e+M \vartheta \\
e & =u_{i, i} \\
\vartheta & =-w_{i, i} \\
w_{i} & =\phi\left(U_{i}-u_{i}\right),
\end{aligned}
$$

where $\sigma_{i j}$ is the stress of soil, $\varepsilon_{i j}$ is the strain tensor of soil, $\delta_{i j}$ is the Kronecker delta, $u_{i}$ and $U_{i}$ are, respectively, the average displacements of the soil and fluid, $w_{i}$ is the penetration displacement of the fluid, $p_{f}$ is the excess pore water pressure, $\lambda$ and $\mu$ are lame constant, $e$ is the volumetric strain of soil skeleton, $\vartheta$ is the fluid volume increment of unit porous media, $\alpha$ and $M$ are Biot's parameters with regard to the saturated porous media compression, and $\phi$ is the porosity of porous media.

The motion equations of porous media can be expressed as

$$
\begin{aligned}
& \mu u_{i, j j}+\left(\lambda+\alpha^{2} M+\mu\right) u_{j, j i}+\alpha M w_{j, j i}=\rho \ddot{u}_{i}+\rho_{f} \ddot{w}_{i}, \\
& \alpha M u_{j, j i}+M w_{j, j i}=\rho_{f} \ddot{u}_{i}+m \ddot{w}_{i}+b_{p} \dot{w}_{i},
\end{aligned}
$$

where $\rho$ and $\rho_{f}$ are, respectively, the density of porous media and the density of fluid, $\rho_{s}$ is the density of soil skeleton, $m=$ $a_{\infty} \rho_{f} / \phi, a_{\infty}$ is the bending coefficient of porous media, $b_{p}=$ $\eta / k, b_{p}$ is the interaction force between soil skeleton and fluid, $\eta$ is the viscosity coefficient of porous medium, and $k$ is the dynamic permeability coefficient of porous medium.

Based on the method of Helmhotlz vector decomposition and Fourier transform, the soil displacement in frequency domain has the following form:

$$
\bar{u}_{i}=\bar{\varphi}_{, i}+e_{i j k} \bar{\psi}_{k, j}
$$

where the superscript " - " expresses Fourier transform from $t$ to $\omega, \bar{\varphi}$ and $\bar{\psi}_{k}(k=1,2,3)$ are, respectively, scalar potential and vector potential of soil displacement in transform domain, $e_{i j k}$ is the Ricci symbol, the vector potential $\bar{\psi}_{k}$ satisfies the regular condition, and

$$
\bar{\varphi}_{i, i}=0 \text {. }
$$

Because there are two kinds of $\mathrm{P}$ wave $\left(\mathrm{P}_{1}\right.$ wave and $\mathrm{P}_{2}$ wave) in saturated soil, (17) can be expressed as

$$
\bar{u}_{i}=\bar{\varphi}_{, i}+e_{i j k} \bar{\psi}_{k, j}=\bar{\varphi}_{f, i}+\bar{\varphi}_{s, i}+e_{i j k} \bar{\psi}_{k, j} \text {, }
$$

where $\bar{\varphi}_{f}$ and $\bar{\varphi}_{s}$ are scalar potential of $\mathrm{P}_{1}$ wave and $\mathrm{P}_{2}$ wave.

According to the analysis of Bonnet $[16,17]$, the pore pressure is as follows:

$$
\bar{p}_{f}=A_{f} \bar{\varphi}_{f, i i}+A_{s} \bar{\varphi}_{s, i i}
$$

where $A_{f}$ and $A_{s}$ are constants determined by Biot's control equation. 
Equations (13), (16), (19), and (20) lead to the following equation:

$$
\begin{aligned}
& {\left[\left(\lambda+2 \mu-\beta_{2} A_{f}\right) \bar{\varphi}_{f, j j}+\beta_{3} \bar{\varphi}_{f}\right]_{, i}} \\
& +\left[\left(\lambda+2 \mu-\beta_{2} A_{s}\right) \bar{\varphi}_{s, j j}+\beta_{3} \bar{\varphi}_{s}\right]_{, i} \\
& +e_{i m l}\left[\mu \bar{\psi}_{l, j j}+\beta_{3} \bar{\psi}_{l}\right]_{, m}=0 .
\end{aligned}
$$

Equation (21) has the following expressions:

$$
\begin{aligned}
\left(\lambda+2 \mu-\beta_{2} A_{f}\right) \bar{\varphi}_{f, j j}+\beta_{3} \bar{\varphi}_{f} & =0, \\
\left(\lambda+2 \mu-\beta_{2} A_{s}\right) \bar{\varphi}_{s, j j}+\beta_{3} \bar{\varphi}_{s} & =0, \\
\mu \bar{\psi}_{i, j j}+\beta_{3} \bar{\psi} & =0,
\end{aligned}
$$

where $\beta_{1}=m \omega^{2}-i b_{p} \omega, \beta_{2}=\alpha-\rho_{f} \omega^{2} / \beta_{1}$, and $\beta_{3}=\rho \omega^{2}-$ $\rho_{f}^{2} \omega^{4} / \beta_{1}$.

Equations (16), (17), (18), and (19) lead to the following equation:

$$
\bar{p}_{f, i i}+\frac{\beta_{1}}{M} \bar{p}_{f}+\left(\alpha \beta_{1}-\rho_{f} \omega^{2}\right) \bar{u}_{i, i}=0 .
$$

Equation (24) can be reduced by substituting (19) and (20) into (22):

$$
\begin{aligned}
& {\left[A_{f} \bar{\varphi}_{f, i i}+\left(\beta_{5} A_{f}-\beta_{4}\right) \bar{\varphi}_{f}\right]_{, j j}} \\
& \quad+\left[A_{s} \bar{\varphi}_{s, i i}+\left(\beta_{5} A_{f}-\beta_{4}\right) \bar{\varphi}_{s}\right]_{, j j}=0 .
\end{aligned}
$$

According to equation (24), the following equations should be satisfied.

$$
\begin{gathered}
A_{f} \bar{\varphi}_{f, i i}+\left(\beta_{5} A_{f}-\beta_{4}\right) \bar{\varphi}_{f}=0, \\
A_{s} \bar{\varphi}_{s, i i}+\left(\beta_{5} A_{s}-\beta_{4}\right) \bar{\varphi}_{s}=0,
\end{gathered}
$$

where $\beta_{4}=\rho_{f} \omega^{2}-\alpha \beta_{1}$ and $\beta_{5}=\beta_{1} / M$.

Equation (26) can be obtained by the integration of (22) and (25):

$$
\begin{aligned}
& A_{f, s}^{2}+\frac{\beta_{2}-(\lambda+2 \mu) \beta_{4}-\beta_{1} \beta_{3}}{\beta_{1} \beta_{4}} A_{f, s}+\frac{(\lambda+2 \mu) \beta_{3}}{\beta_{1} \beta_{4}} \\
& \quad=0 .
\end{aligned}
$$

Then, (22) leads to the following Helmholtz equations:

$$
\begin{aligned}
\nabla^{2} \bar{\varphi}_{f}+k_{f}^{2} \bar{\varphi}_{f} & =0, \\
\nabla^{2} \bar{\varphi}_{s}+k_{s}^{2} \bar{\varphi}_{s} & =0, \\
\nabla^{2} \bar{\psi}+k_{t}^{2} \bar{\psi} & =0,
\end{aligned}
$$

where $k_{f}^{2}=\left(\beta_{5} A_{f}-\beta_{4}\right) / A_{f}, k_{s}^{2}=\left(\beta_{5} A_{s}-\beta_{4}\right) / A_{s}, k_{t}^{2}=\beta_{3} / \mu$, $k_{f}, k_{s}$, and $k_{t}$ are complex wave numbers of $\mathrm{P}_{1}$ wave, $\mathrm{P}_{2}$ wave, and $S$ wave, and $\bar{\psi}$ is vector potential of shear wave. In order to guarantee the attenuation of the body waves, $\operatorname{Im}\left(k_{f}\right), \operatorname{Im}\left(k_{s}\right)$, and $\operatorname{Im}\left(k_{t}\right)$ should be nonpositive. Because the speed of $\mathrm{P}_{1}$ wave is larger than that of $\mathrm{P}_{2}$ wave, inequality $\operatorname{Re}\left(k_{f}\right) \leq \operatorname{Re}\left(k_{s}\right)$ should always hold. Detailed derivation of above poroelastic model can be found in Lu et al. [14].
3.2. The Wave Field Solution of Saturated Soil under Rayleigh Surface Wave. Assuming that the Rayleigh wave is two-dimensional inhomogeneous plane wave, the twodimensional Helmholtz equations of saturated soil wave functions in the frequency domain can be obtained by the decoupling control equations for Biot's theory in Cartesian coordinate. Consider

$$
\begin{aligned}
\nabla_{\perp}^{2} \bar{\varphi}_{f}+k_{f}^{2} \bar{\varphi}_{f} & =0, \\
\nabla_{\perp}^{2} \bar{\varphi}_{s}+k_{s}^{2} \bar{\varphi}_{s} & =0, \\
\nabla_{\perp}^{2} \bar{\psi}+k_{t}^{2} \bar{\psi} & =0,
\end{aligned}
$$

where $\varphi_{f}, \varphi_{s}$, and $\bar{\psi}$ are the potential functions for $\mathrm{P}_{1}$ wave, $\mathrm{P}_{2}$ wave, and $\mathrm{S}$ wave of the saturated half space and $\nabla_{\perp}^{2}=\partial^{2} / \partial x^{2}+\partial^{2} / \partial^{2} z$ is the Laplacian operator in the twodimensional Cartesian coordinate system. The displacements and the pore pressure of the pore fluid can be expressed as

$$
\begin{aligned}
& u_{x}=\frac{\partial \bar{\varphi}_{f}}{\partial x}+\frac{\partial \bar{\varphi}_{s}}{\partial x}+\frac{\partial \bar{\psi}}{\partial z}, \\
& u_{z}=\frac{\partial \bar{\varphi}_{f}}{\partial z}+\frac{\partial \bar{\varphi}_{s}}{\partial z}+\frac{\partial \bar{\psi}}{\partial x}, \\
& p_{f}=-A_{f} k_{f}^{2} \bar{\varphi}_{f}-A_{s} k_{s}^{2} \bar{\varphi}_{s} .
\end{aligned}
$$

Rayleigh surface wave is determined by the following potential function:

$$
\begin{aligned}
& \bar{\varphi}_{f}(x, z) e^{i \omega t}=A_{f}^{R}\left[e^{-i k_{r} x-i k_{f} n_{f} z}\right] e^{i \omega t}, \\
& \bar{\varphi}_{s}(x, z) e^{i \omega t}=A_{s}^{R}\left[e^{-i k_{r} x-i k_{s} n_{s} z}\right] e^{i \omega t}, \\
& \bar{\psi}(x, z) e^{i \omega t}=A_{t}^{R}\left[e^{-i k_{r} x-i k_{t} n_{t} z}\right] e^{i \omega t},
\end{aligned}
$$

where $A_{f}^{R}, A_{s}^{R}$, and $A_{t}^{R}$ are, respectively, the wave function amplitude of $\mathrm{P}_{1}$ wave, $\mathrm{P}_{2}$ wave, and $\mathrm{S}$ wave in Rayleigh wave and $k_{f}, k_{s}$, and $k_{t}$ are the complex wave number of the Rayleigh surface waves, respectively; $n_{f}, n_{s}$, and $n_{t}$ are the complex direction cosines of $\mathrm{P}_{1}$ wave, $\mathrm{P}_{2}$ wave, and $\mathrm{S}$ wave.

The following can be reduced by substituting the potential function into the two-dimensional Helmholtz equations:

$$
\begin{gathered}
n_{f}^{2} k_{f}^{2}+k_{r}^{2}=k_{f}^{2}, \\
n_{s}^{2} k_{s}^{2}+k_{r}^{2}=k_{s}^{2}, \\
n_{t}^{2} k_{t}^{2}+k_{r}^{2}=k_{t}^{2} .
\end{gathered}
$$

The following boundary conditions for a fully permeable surface are as follows:

$$
\begin{gathered}
\bar{\sigma}_{z z}(x, 0)=0, \\
\bar{\sigma}_{z x}(x, 0)=0, \\
\bar{p}_{f}(x, 0)=0 .
\end{gathered}
$$

The displacements, stresses, and pore pressure can be obtained based on the equations (28)-(32). Three equations 
about $A_{f}^{R}, A_{s}^{R}$ and $A_{t}^{R}$ can be reduced based on the boundary conditions. The complex Rayleigh equation of saturated soil can be calculated by the conditions of the equation coefficient ranks as the zero. In order to guarantee the attenuation of the body waves, $\operatorname{Im}\left(k_{r}\right)$ should be nonpositive; the roots of $n_{f}, n_{s}$, and $n_{t}$ satisfy $\operatorname{Re}\left(k_{i} n_{i}\right) \geq 0$ and $\operatorname{Im}\left(k_{i} n_{i}\right) \leq 0, i=f, s, t$.

3.3. Floquet Transform Method. The displacement amplitude at the bottom of different piers of the periodic structure varies with spatial locations in the action of Rayleigh waves, and the displacive phase transition between adjacent piers is uncertain. That is to say, the dynamic response of periodic viaduct structure is caused by a series of nonuniform waves; it is impossible to employ the periodic condition of the viaduct to simplify its dynamic analysis directly. Therefore, the Floquet transform method is introduced to convert the spatial nonuniform waves to harmonic waves in wave number domain. For periodic viaduct in-plane vibration, considering the lattice vector cycle of adjacent lattice points in the one-dimensional direction to be $L$, the lattice vector $R$ can be expressed as $\mathbf{R}=n L e$ according to literature [18], where $e$ is basis vector of the one-dimensional direction. If $f(\mathbf{R})=f(n L)$ is the discrete functions in spatial domain of one-dimensional vector, the Floquet transform and inverse transform can be defined as follows [19]:

$$
\begin{gathered}
F(f(n L))=\widehat{f}(\kappa)=\sum_{n=-\infty}^{+\infty} f(n L) e^{i \kappa n L}, \\
f(n L)=\frac{L}{2 \pi} \int_{-\pi / L}^{\pi / L} \widehat{f}(\kappa) e^{-i \kappa n L} d \kappa,
\end{gathered}
$$

where $\kappa$ is the wave number of lattice waves and the superscript " " " means wave number domain.

Thus, the Floquet transform of discrete spatial sequence function $f[(m+n) L]$ can be expressed as

$$
\begin{aligned}
F(f[(m+n) L]) & =\widehat{f}^{(n)}(\kappa) \\
& =\sum_{m=-\infty}^{+\infty} f[(m+n) L] e^{i \kappa m L} \\
& =e^{-i \kappa n L} \widehat{f}(\kappa) .
\end{aligned}
$$

Considering the solution of the viaduct in wave field due to Rayleigh wave, the Fourier transform between time $t$ and frequency $\omega$ is defined as follows:

$$
\begin{aligned}
& \bar{f}(\omega)=\int_{-\infty}^{+\infty} f(t) e^{-i \omega t} d t, \\
& f(t)=\frac{1}{2 \pi} \int_{-\infty}^{+\infty} \bar{f}(\omega) e^{i \omega t} d \omega,
\end{aligned}
$$

where the superscript "-" indicates the function is to be in frequency domain.

With (34) and (35), the dynamic response in time domain can be transformed into harmonic wave with amplitude $\hat{\bar{f}}(k)$ in frequency wave number domain.

\section{Dynamic Response of In-Plane Vibration of the Periodic Viaduct}

4.1. Dynamic Response in Frequency Wave Number Domain. The displacement vector $\hat{\overline{\mathbf{q}}}_{d}^{(n)}\left(\kappa, L_{d}\right)$ at the bottom of viaduct pier due to Rayleigh waves can be deduced based on the Floquet transform of discrete spatial sequence function. Through the transfer matrix of pier, the displacement vector $\hat{\overline{\mathbf{q}}}_{d}^{(n)}\left(\kappa, 0^{+}\right)$and the internal force vector at the top of pier can be derived as follows:

$$
\begin{aligned}
\hat{\overline{\mathbf{q}}}_{d}^{(n)}\left(\kappa, 0^{+}\right)= & \mathbf{C}_{d} \widehat{\overline{\mathbf{f}}}_{d}^{(n)}\left(\kappa, 0^{+}\right) \\
& +\mathbf{T}_{q q}^{(d)-1}\left(L_{d}\right) \widehat{\overline{\mathbf{q}}}_{d}^{(n)}\left(\kappa, L_{d}\right),
\end{aligned}
$$

where $\quad \mathbf{C}_{d}=-\mathbf{T}_{q q}^{(d)-1}\left(L_{d}\right) \mathbf{T}_{q f}^{(d)}\left(L_{d}\right), \quad \mathbf{T}^{(d)}\left(L_{d}\right)=$

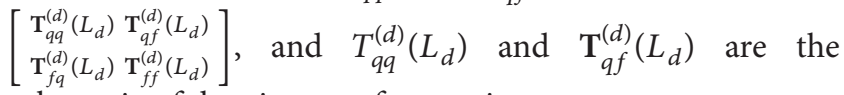
submatrix of the pier transfer matrix.

Equation (10) is expressed in matrix form as

$$
\widehat{\overline{\mathbf{f}}}_{d}^{(n)}\left(\kappa, 0^{+}\right)=\mathbf{E}_{l}^{(a)} \hat{\overline{\mathbf{f}}}_{b}^{(n)}\left(\kappa, 0^{-}\right)+\mathbf{E}_{r}^{(a)} \hat{\overline{\mathbf{f}}}_{b}^{(n)}\left(\kappa, 0^{+}\right),
$$

where $\mathbf{E}_{l}^{(a)}=\left[\begin{array}{ccc}0 & 1 & 0 \\ -1 & 0 & 0 \\ 0 & 0 & -1\end{array}\right]$ and $\mathbf{E}_{r}^{(a)}=\left[\begin{array}{ccc}0 & -1 & 0 \\ 1 & 0 & 0 \\ 0 & 0 & 1\end{array}\right]$.

The following equation can be obtained by substituting (37) into (36):

$$
\begin{aligned}
\hat{\overline{\mathbf{q}}}_{d}^{(n)}\left(\kappa, 0^{+}\right)= & E_{l}^{(b)} \hat{\overline{\mathbf{f}}}_{b}^{(n)}\left(\kappa, 0^{-}\right)+E_{r}^{(b)} \hat{\overline{\mathbf{f}}}_{b}^{(n)}\left(\kappa, 0^{+}\right) \\
& +\mathbf{T}_{q q}^{(d)-1}\left(L_{d}\right) \hat{\overline{\mathbf{q}}}_{d}^{(n)}\left(\kappa, L_{d}\right)
\end{aligned}
$$

where $\mathbf{E}_{l}^{(b)}=\mathbf{C}_{d} \mathbf{E}_{l}^{(a)}$ and $\mathbf{E}_{r}^{(b)}=\mathbf{C}_{d} \mathbf{E}_{r}^{(a)}$.

The internal force vectors of the left and the right beams in the $n$th span unit are deduced and are obtained as follows based on (6), (7), and (10) and (6), (8), and (11):

$$
\begin{aligned}
\hat{\overline{\mathbf{f}}}_{b}^{(n)}\left(\kappa, 0^{-}\right)= & \mathbf{J}_{l l} \hat{\overline{\mathbf{q}}}_{b}^{(n)}\left(\kappa, 0^{-}\right)+\mathbf{J}_{l r} \hat{\overline{\mathbf{q}}}_{b}^{(n)}\left(\kappa, 0^{+}\right) \\
& +\mathbf{J}_{l d} \hat{\overline{\mathbf{q}}}_{d}^{(n)}\left(\kappa, 0^{+}\right), \\
\widehat{\overline{\mathbf{f}}}_{b}^{(n)}\left(\kappa, 0^{+}\right)= & \mathbf{J}_{r l} \hat{\overline{\mathbf{q}}}_{b}^{(n)}\left(\kappa, 0^{-}\right)+\mathbf{J}_{r r} \hat{\overline{\mathbf{q}}}_{b}^{(n)}\left(\kappa, 0^{+}\right) \\
& +\mathbf{J}_{r d} \hat{\overline{\mathbf{q}}}_{d}^{(n)}\left(\kappa, 0^{+}\right),
\end{aligned}
$$

$$
\begin{aligned}
& \text { where } \mathbf{J}_{l l}=\left[\begin{array}{ccc}
-\left(k_{t}^{(t)}+k_{l}^{(s)}\right) & 0 & 0 \\
0 & -\left(k_{t}^{(s)}+k_{l}^{(t)}\right) & 0 \\
0 & 0 & k_{t}^{(b)}+k_{l}^{(b)}
\end{array}\right], \mathbf{J}_{l r}=\left[\begin{array}{ccc}
k_{t}^{(t)} & 0 & 0 \\
0 & k_{t}^{(s)} & 0 \\
0 & 0 & -k_{t}^{(b)}
\end{array}\right] \text {, } \\
& \mathbf{J}_{r r}=\left[\begin{array}{ccc}
k_{t}^{(t)}+k_{r}^{(s)} & 0 & 0 \\
0 & k_{t}^{(s)}+k_{r}^{(t)} & 0 \\
0 & 0 & -\left(k_{t}^{(b)}+k_{r}^{(b)}\right)
\end{array}\right], \mathbf{J}_{l d}=\left[\begin{array}{ccc}
0 & k_{l}^{(s)} & 0 \\
k_{l}^{(t)} & 0 & 0 \\
0 & 0 & k_{l}^{(b)}
\end{array}\right], \mathbf{J}_{r l}= \\
& {\left[\begin{array}{ccc}
-k_{t}^{(t)} & 0 & 0 \\
0 & -k_{t}^{(s)} & 0 \\
0 & 0 & k_{t}^{(b)}
\end{array}\right] \text {, and } \mathbf{J}_{r d}=\left[\begin{array}{ccc}
0 & -k_{r}^{(s)} & 0 \\
-k_{r}^{(t)} & 0 & 0 \\
0 & 0 & -k_{r}^{(b)}
\end{array}\right] \text {. }}
\end{aligned}
$$


Substituting (38) into (39), the state vector of the left side and right side of horizontal beam-beam spring can be expressed as

$$
\left\{\begin{array}{l}
\hat{\overline{\mathbf{q}}}_{b}^{(n)}\left(\kappa, 0^{+}\right) \\
\widehat{\overline{\mathbf{f}}}_{b}^{(n)}\left(\kappa, 0^{+}\right)
\end{array}\right\}=\mathbf{S}_{J}\left\{\begin{array}{l}
\widehat{\overline{\mathbf{q}}}_{b}^{(n)}\left(\kappa, 0^{-}\right) \\
\widehat{\overline{\mathbf{f}}}_{b}^{(n)}\left(\kappa, 0^{-}\right)
\end{array}\right\}+\mathbf{A}^{-1} \widehat{\overline{\mathbf{R}}}^{(n)}(\kappa)
$$

where $\mathbf{A}=\left[\begin{array}{cc}\mathbf{J}_{l r} & \mathbf{J}_{l d} \mathbf{E}_{r}^{(b)} \\ \mathbf{J}_{r r} & \mathbf{J}_{r d} \mathbf{E}_{r}^{(b)}-\mathbf{I}\end{array}\right], \mathbf{B}=\left[\begin{array}{cc}-\mathbf{J}_{l l} & \mathbf{I}-\mathbf{J}_{l d} \mathbf{E}_{l}^{(b)} \\ -\mathbf{J}_{r l} & -\mathbf{J}_{r d} \mathbf{E}_{l}^{(b)}\end{array}\right], \widehat{\overline{\mathbf{R}}}^{(n)}(\kappa)=$ $\left[\begin{array}{c}-\mathbf{J}_{l d} \mathbf{T}_{q q}^{(d)-1}\left(L_{d}\right) \hat{\overline{\mathbf{q}}}_{d}^{(n)}\left(\kappa, L_{d}\right) \\ -\mathbf{J}_{r d} \mathbf{T}_{q q}^{(d)-1}\left(L_{d}\right) \hat{\overline{\mathbf{q}}}_{d}^{(n)}\left(\kappa, L_{d}\right)\end{array}\right]$, and $\mathbf{S}_{J}=\mathbf{A}^{-1} B$.

The state vector of the left beam and right beam can be obtained by the solution of (40) and horizontal beam transfer matrix $\mathbf{T}^{(b)}\left(L_{b}\right)$ :

$$
\begin{aligned}
\widehat{\bar{\psi}}_{b}^{(n)}\left(\kappa, \frac{L_{b}}{2}\right) \\
=\mathbf{T}^{(b)}\left(\frac{L_{b}}{2}\right) \mathbf{S}_{J} \mathbf{T}^{(b)}\left(\frac{L_{b}}{2}\right) \widehat{\bar{\psi}}_{b}^{(n)}\left(\kappa,-\frac{L_{b}}{2}\right) \\
+\mathbf{T}^{(b)}\left(\frac{L_{b}}{2}\right) \mathbf{A}^{-1} \widehat{\overline{\mathbf{R}}}^{(n)}(\kappa) .
\end{aligned}
$$

According to Bloch's theory [19], the state vector of periodic structure satisfies the following equation in wave number domain:

$$
\widehat{\bar{\psi}}_{b}^{(n)}\left(\kappa, \frac{L_{b}}{2}\right)=e^{-i \kappa L} \widehat{\bar{\psi}}_{b}^{(n)}\left(\kappa,-\frac{L_{b}}{2}\right) .
$$

Using (41) and (42), the state vector of the left beam in the periodic element can be obtained:

$$
\begin{aligned}
& {\left[\mathbf{T}^{(b)}\left(\frac{L_{b}}{2}\right) \mathbf{S}_{J} \mathbf{T}^{(b)}\left(\frac{L_{b}}{2}\right)-e^{-i \kappa L} \mathbf{I}_{6 \times 6}\right] \widehat{\bar{\psi}}_{b}^{(n)}\left(\kappa,-\frac{L_{b}}{2}\right)} \\
& =\mathbf{T}^{(b)}\left(\frac{L_{b}}{2}\right) \mathbf{A}^{-1} \widehat{\overline{\mathbf{R}}}^{(n)}(\kappa) .
\end{aligned}
$$

Excluding the vibration of piers caused by the Rayleigh waves, $\widehat{\overline{\mathbf{R}}}^{(n)}(\kappa)$ is zero in (43); thus, the characteristic equation of in-plane vibration for the periodic viaduct can be obtained:

$$
\begin{aligned}
& {\left[\mathbf{T}^{(b)}\left(\frac{L_{b}}{2}\right) \mathbf{S}_{J} \mathbf{T}^{(b)}\left(\frac{L_{b}}{2}\right)-e^{-i \kappa_{\alpha} L} \mathbf{I}_{6 \times 6}\right] \widehat{\bar{\psi}}_{b}^{(n)}\left(\kappa,-\frac{L_{b}}{2}\right)} \\
& \quad=0
\end{aligned}
$$

where $\kappa_{\alpha}$ is complex wave number of wave propagation in periodic viaduct of which the real part means the phase transition of lattice wave and the imaginary part indicates wave decay in periodic viaduct.

4.2. Dynamic Response in Spatial Domain. The state vector of the left beam can be gotten in the frequency wave number domain based on (43). The solution $\bar{\psi}_{b}^{(n)}\left(n L_{b},-L_{b} / 2\right)$ in the
TABLE 1: The parameters of the piers and beams.

\begin{tabular}{lc}
\hline Young's modulus of pier $E_{d}(\mathrm{~Pa})$ & $2.8 \times 10^{10}$ \\
Poisson's ratio $v_{d}$ & 0.3 \\
Pier density $\rho_{d}\left(\mathrm{~kg} / \mathrm{m}^{3}\right)$ & $3.0 \times 10^{3}$ \\
Pier height $L_{d}(\mathrm{~m})$ & 7.5 \\
Pier radius $R_{d}(\mathrm{~m})$ & 0.3 \\
Young's modulus of beam $E_{b}(\mathrm{~Pa})$ & $2.8 \times 10^{10}$ \\
Beam density $\rho_{b}\left(\mathrm{~kg} / \mathrm{m}^{3}\right)$ & $3.5 \times 10^{3}$ \\
Span $L_{b}(\mathrm{~m})$ & 10.0 \\
Cross-sectional height of beam $h_{b}(\mathrm{~m})$ & 0.3 \\
Cross-sectional width of beam $w_{b}(\mathrm{~m})$ & 2.0 \\
\hline
\end{tabular}

TABLE 2: The spring stiffness of beam-beam and beam-pier.

\begin{tabular}{lcc}
\hline \multirow{3}{*}{ Spring stiffness of beam-beam } & $k_{t t}(\mathrm{~N} / \mathrm{m})$ & $5.0 \times 10^{8}$ \\
& $k_{t s}(\mathrm{~N} / \mathrm{m})$ & $5.0 \times 10^{8}$ \\
& $k_{t b}(\mathrm{~N} \cdot \mathrm{m} / \mathrm{rad})$ & $1.0 \times 10^{8}$ \\
\hline \multirow{3}{*}{ Spring stiffness of left beam-pier } & $k_{l t}(\mathrm{~N} / \mathrm{m})$ & $2.0 \times 10^{8}$ \\
& $k_{l s}(\mathrm{~N} / \mathrm{m})$ & $2.0 \times 10^{8}$ \\
& $k_{l b}(\mathrm{~N} \cdot \mathrm{m} / \mathrm{rad})$ & $0.5 \times 10^{8}$ \\
\hline \multirow{3}{*}{ Spring stiffness of right beam-pier } & $k_{r t}(\mathrm{~N} / \mathrm{m})$ & $2.0 \times 10^{8}$ \\
& $k_{r s}(\mathrm{~N} / \mathrm{m})$ & $2.0 \times 10^{8}$ \\
& $k_{r b}(\mathrm{~N} \cdot \mathrm{m} / \mathrm{rad})$ & $0.5 \times 10^{8}$ \\
\hline
\end{tabular}

frequency spatial domain can be obtained by means of the inverse Floquet transform. Consider

$$
\begin{gathered}
\bar{\psi}_{b}^{(n)}\left(n L_{b},-\frac{L_{b}}{2}\right)=\frac{L_{b}}{2 \pi} \int_{-\pi / L_{b}}^{\pi / L_{b}} \widehat{\bar{\psi}}_{b}^{(n)}\left(\kappa,-\frac{L_{b}}{2}\right) d \kappa \\
=\frac{L_{b}}{2 \pi} \int_{-\pi / L_{b}}^{\pi / L_{b}} \widehat{\bar{\psi}}_{b}\left(\kappa,-\frac{L_{b}}{2}\right) e^{-i n \kappa L_{b}} d \kappa .
\end{gathered}
$$

\section{Numerical Results}

As for the periodic viaduct shown in Figure 1, considering the cross section of horizontal beam to be rectangular and that of pier to be circular, the parameters of the pier and beams given in Table 1 and the spring stiffness of beam-beam and beam-pier given in Table 2 are employed to study the dynamic response under Rayleigh surface wave.

In the paper, Rayleigh wave is adopted as the vibration source in saturated soil to simulate seismic wave field. The center coordinate $\left(x_{s}, y_{s}\right.$, and $\left.z_{s}\right)$ of the unit circle load is $(0.0 \mathrm{~m}, 20.0 \mathrm{~m}$, and $10.0 \mathrm{~m})$, the radius $R$ is $0.5 \mathrm{~m}$, and the load amplitude $F_{z}$ is $1.0 \mathrm{~N}$. The parameters of saturated soil are as follows: $\mu=2.0 \times 10^{7} \mathrm{~N} / \mathrm{m}^{2}, a_{\infty}=2.0, \lambda=4.0 \times 10^{7} \mathrm{~N} / \mathrm{m}^{2}$, $\alpha=0.97, b_{p}=1.94 \times 10^{6} \mathrm{~kg} / \mathrm{m}^{3} \cdot \mathrm{s}, M=2.4 \times 10^{8} \mathrm{~N} / \mathrm{m}^{2}$, $\phi=0.4, \rho_{s}=2.0 \times 10^{3} \mathrm{~kg} / \mathrm{m}^{3}$, and $\rho_{f}=1.0 \times 10^{3} \mathrm{~kg} / \mathrm{m}^{3}$.

5.1. Energy Band Analysis of the Periodic Structure. The transfer matrix for each span of the periodic viaduct is a $6 \times 6$ matrix in (44). Therefore, there exist three kinds of characteristic waves corresponding to axial compression, transverse shear, and bending vibration, respectively, in the periodic 

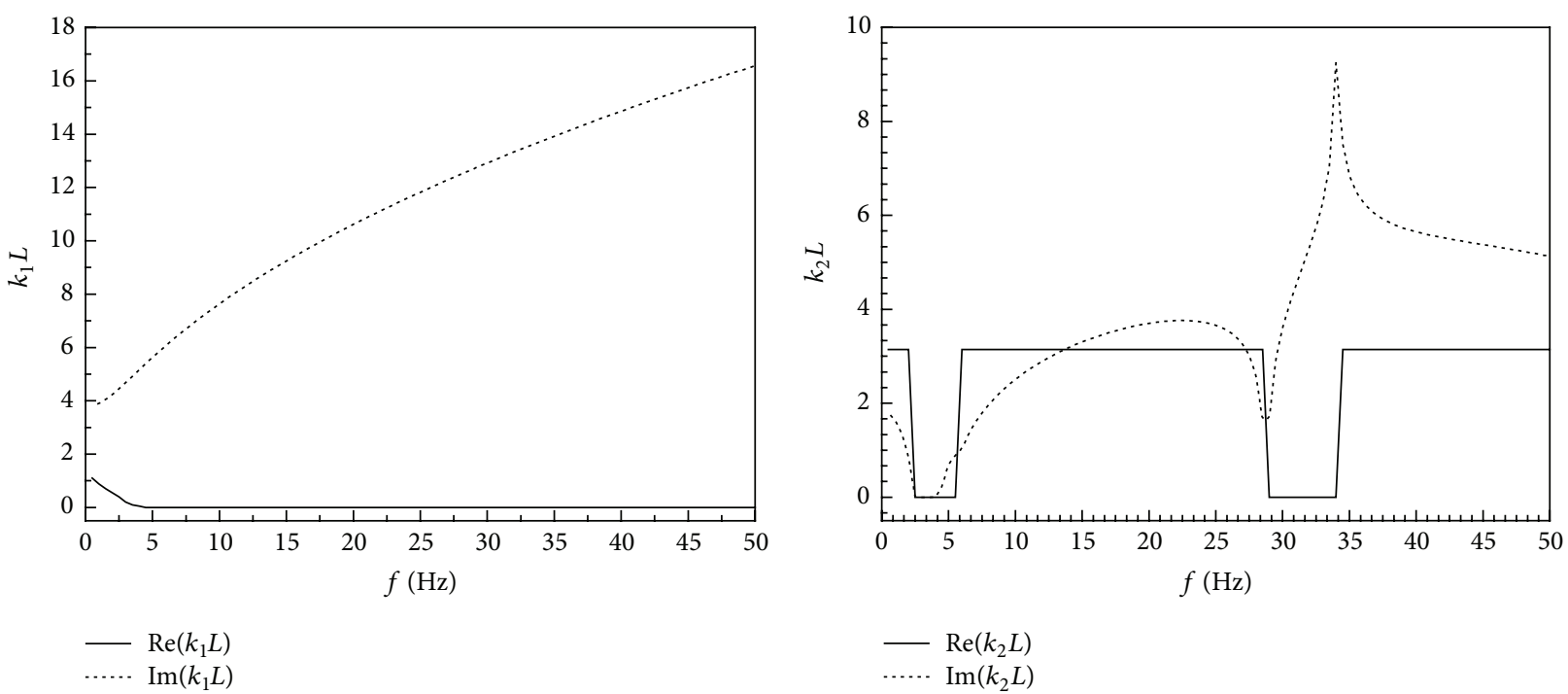

(a)

(b)

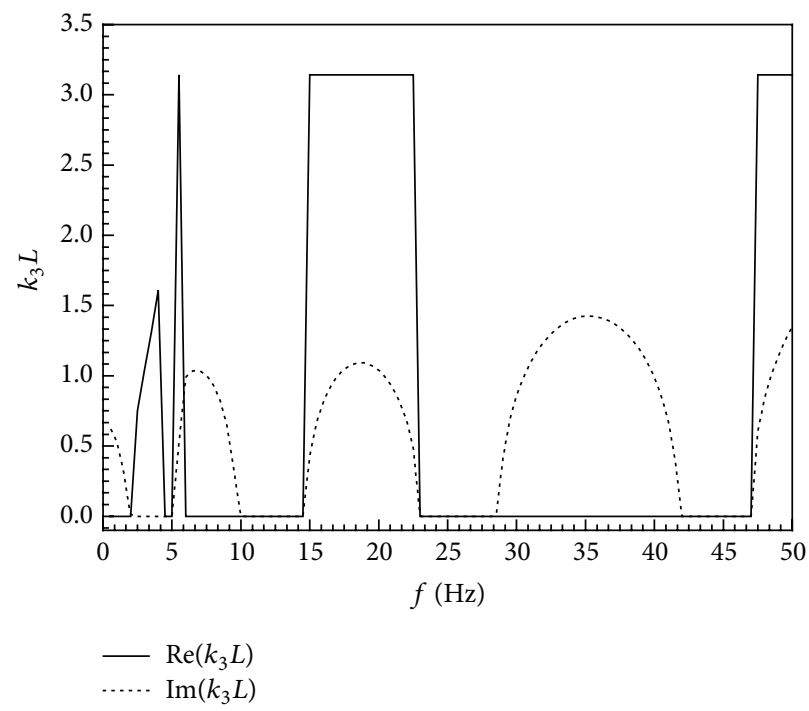

(c)

FIGURE 4: The energy bands for the characteristic waves of the periodic viaduct undergoing in-plane vibration. (a) The wave number for the first kind of wave. (b) The wave number for the second kind of wave. (c) The wave number for the third kind of wave.

viaduct for the in-plane vibration [19]; the real part represents the phase transformation of the wave propagation in the structure, and the imaginary part represents the attenuation of the propagating wave.

Figure 4 shows the energy bands of the three kinds of characteristic waves propagating in the periodic viaduct for in-plane vibration. The distribution of their energy bands varies with different frequencies. The imaginary part of the first kind and the second kind of waves is larger, which indicates the first kind and second kind of waves cannot propagate far in the periodic viaduct, and vibration waves decay rapidly. The imaginary part of the third kind of wave shows the alternating pass band and band gap at different frequencies and is smaller than that of above two kinds of waves, which indicates the wave mainly propagating in the viaduct vibration is the third kind.
5.2. Dynamic Response of the Periodic Structure under Nonuniform Load. Using Rayleigh surface wave as vibration source, according to the solution of literature [14], the displacement vector at the bottom of pier for the periodic viaduct can be obtained in the frequency domain. By means of the Floquet transform of (1), the displacement vector at the bottom of pier can be obtained in the frequency wave number domain, wherein the infinite sequence summation of (1) is expressed in finite terms with the sum number 101 in the paper. The inverse Floquet transform of (2) from the wave number domain to spatial domain can be realized by numerical integration method.

Figures 5 and 6 show dimensionless axial amplitude $\left(u_{x} \mu R / F_{z}\right)$ and tangential displacement amplitude $\left(u_{z} \mu R / F_{z}\right)$ of the horizontal beam end of the periodical viaduct when 


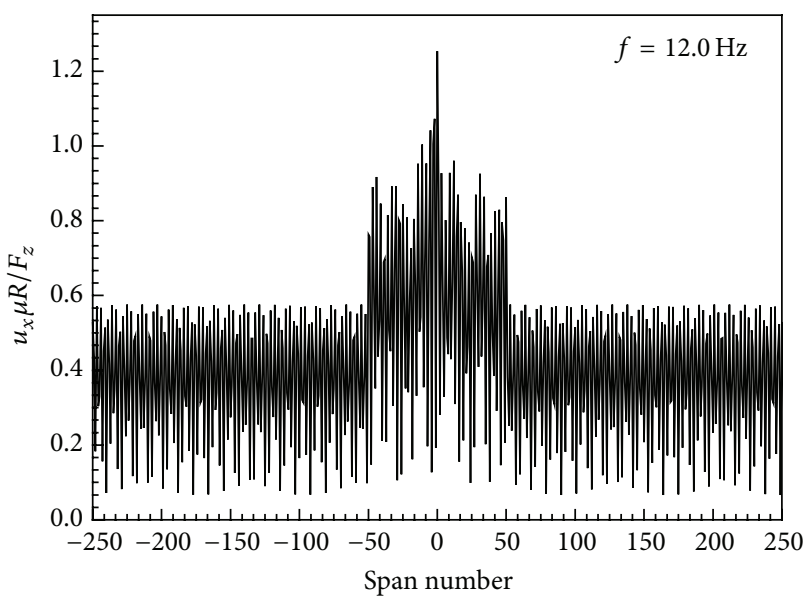

(a)

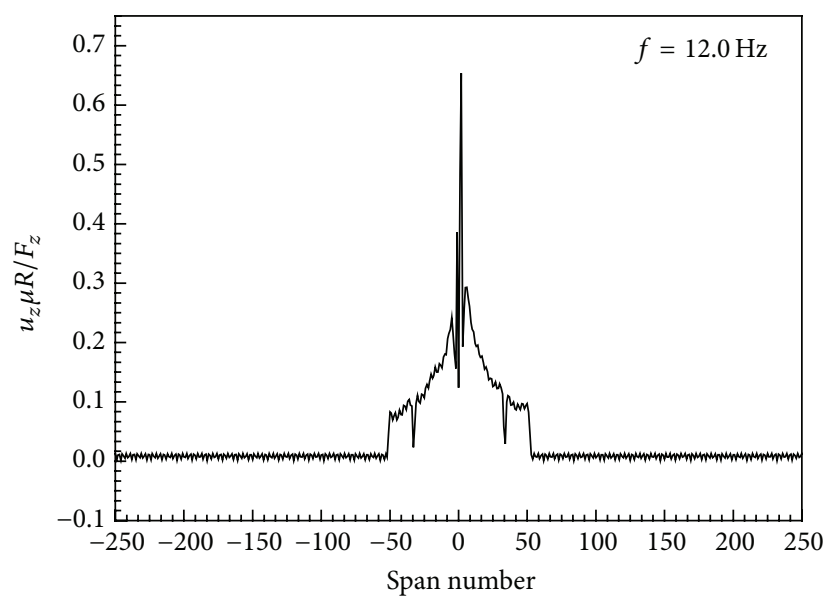

(b)

Figure 5: Dynamic response of the periodic viaduct when the frequency of the excitation source is equal to $12.0 \mathrm{~Hz}$ (pass band). (a) The amplitude of the longitudinal displacement. (b) The amplitude of the transverse displacement.

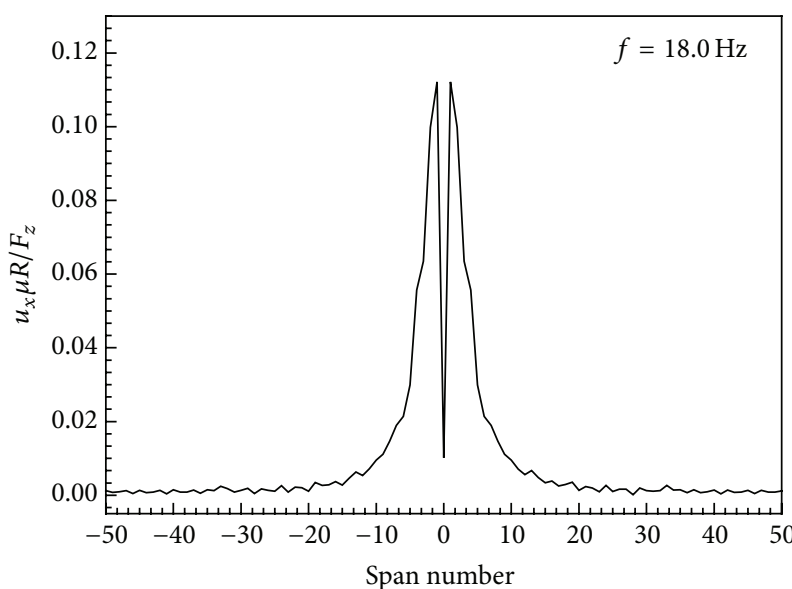

(a)

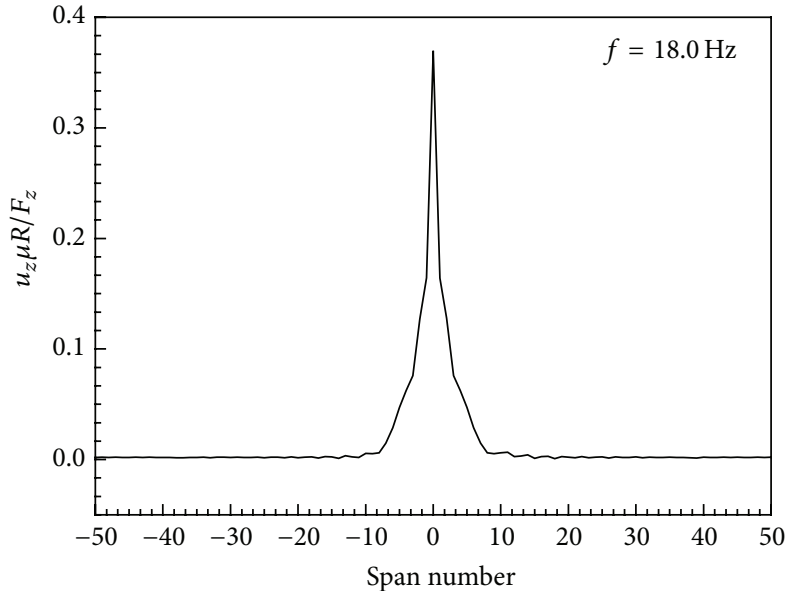

(b)

Figure 6: Dynamic response of the periodic viaduct when the frequency of the excitation source is equal to $12.0 \mathrm{~Hz}$ (stop band). (a) The amplitude of the longitudinal displacement. (b) The amplitude of the transverse displacement.

the frequency of vibration source is equal to $12.0 \mathrm{~Hz}$ and $18.0 \mathrm{~Hz}$, respectively.

As shown in Figure 5(a), when the frequency of vibration source is equal to $12.0 \mathrm{~Hz}$, the axial displacement of the beam at a distance of 100 spans $(-50,50)$ from the vibration source decays rapidly, while it decays slowly when the distance is over 100 spans. The main reason is the fact that the vibration of the viaduct is mainly caused by external vibration source within the range of 100 spans, but, when the distance to the vibration source is more than a certain range (outside of 100 spans), due to the load frequency of vibration source within the pass band of periodic viaduct energy band, the characteristic wave can propagate in periodic structure and cannot decay, which causes vibrations of structure far from the vibration source. However, as shown in Figure 5(b), the transverse displacement far away from the vibration source (outside of 100 spans) attenuates rapidly. Therefore, it can be seen that the wave propagation with vibration source within the pass band of viaduct structure is mainly the third kind of wave.

As shown in Figure 6, when the frequency of vibration source is equal to $18.0 \mathrm{~Hz}$, the dynamic response at the range of 20 spans $(-10,10)$ away from the excitation source is larger, while the longitudinal displacement and transverse displacement decay rapidly when the structure is far away from the excitation source. The main reason is the fact that the vibration attenuates rapidly and propagates in a short distance when the frequency of excitation source is in the range of band gap of periodic structure energy band.

\section{Conclusion}

In the paper, combining the Floquet transform and transfer matrix method, using the periodicity of viaduct structure, 
the in-plane vibration response of periodic viaduct on saturated soil subjected to Rayleigh surface wave excitation is analyzed. Firstly, the periodical structure model of beambeam and beam-pier connected by springs is established in the frequency wave number domain, and the transfer matrix of spring junctions, horizontal beams, and pier are deduced. Then the characteristic equation and the vibration control equations of viaduct structure are established. Finally, the solution of spatial domain is obtained by the inverse Floquet transform. Using numerical examples, the propagation characteristics of waves and the vibration characteristics of periodical viaduct subjected to Rayleigh wave are discussed. And the numerical results show the following:

(1) There are three kinds of wave which existed in periodic viaduct. The first kind of wave decays rapidly, the second kind of wave propagates only in a limited frequency domain, and the third kind of wave propagates in the pass band and decays slowly.

(2) The vibration characteristic of periodic structure far from the vibration source of Rayleigh wave is determined by the characteristic wave propagating in the structure. When the frequency of the vibration source is in the range of pass band, the characteristic wave in the structure will decay slowly and propagate farther. Therefore, there exists structure vibration far away from the vibration source.

(3) When the frequency of the incident Rayleigh wave is in the range of band gap of periodic viaduct, the dynamic response of viaduct structure only exists in the vicinity of vibration source, and vibration waves decay rapidly, which cannot spread along the structure.

\section{Conflict of Interests}

The authors declare that there is no conflict of interests regarding the publication of this paper.

\section{Acknowledgments}

This paper was supported by the National Natural Science Foundation (Project 51269021), the Jiangxi Province Science and Technology Project (20133ACB20006, 20114BAB216010, and 20122BBG70174), and the Jiangxi Provincial Education Department of Science and Technology Project (GJJ14755 and GJJ14756) in China.

\section{References}

[1] M. M. Sigalas and E. N. Economou, "Elastic and acoustic wave band structure," Journal of Sound and Vibration, vol. 158, no. 2, pp. 377-382, 1992.

[2] M. S. Kushwaha, P. Halevi, L. Dobrzynski, and B. DjafariRouhani, "Acoustic band structure of periodic elastic composites," Physical Review Letters, vol. 71, no. 13, pp. 2022-2025, 1993.

[3] A. D. Kiureghian and A. Neuenhofer, "Response spectrum method for multi-support seismic excitations," Earthquake
Engineering \& Structural Dynamics, vol. 21, no. 8, pp. 713-740, 1992.

[4] M. P. Singh and G. O. Maldonado, "An improved response spectrum method for calculating seismic design response: classically damped structures," Earthquake Engineering \& Structural Dynamics, vol. 20, no. 7, pp. 621-635, 1991.

[5] L. Chen, Seismic response analysis on a long span hybrid girder continuous rigid-frame bridge [M.S. thesis], Central South University, Changsha, China, 2013.

[6] A. S. Nazmy and A. M. Abdel-Ghaffar, "Non-linear earthquakeresponse analysis of long-span cable-stayed bridges: theory," Earthquake Engineering \& Structural Dynamics, vol. 19, no. 1, pp. 45-62, 1990.

[7] J. T. Du, Seismic analysis of long-span continuous rigid frame bridge [M.S. thesis], Chang'an University, Xi'an, China, 2013.

[8] T. J. Ingham, S. Rodriguez, and M. Nader, "Nonlinear analysis of the Vincent Thomas Bridge for seismic retrofit," Computers and Structures, vol. 64, no. 5-6, pp. 1221-1238, 1997.

[9] J. H. Lin, Y. H. Zhang, Q. S. Li, and F. W. Williams, "Seismic spatial effects for long-span bridges, using the pseudo excitation method," Engineering Structures, vol. 26, no. 9, pp. 1207-1216, 2004.

[10] J. J. Wang, Study on the dynamic response of curved continuous bridges under spatial seismic excitation [M.S. thesis], Beijing University of Technology, Beijing, China, 2013.

[11] K. Soyluk, "Comparison of random vibration methods for multi-support seismic excitation analysis of long-span bridges," Engineering Structures, vol. 26, no. 11, pp. 1573-1583, 2004.

[12] M. A. Biot, "Theory of propagation of elastic waves in a fluidsaturated porous solid. II. Higher frequency range," Journal of the Acoustical Society of America, vol. 28, no. 2, pp. 179-191, 1956.

[13] M. A. Biot, "Mechanics of deformation and acoustic propagation in porous media," Journal of Applied Physics, vol. 33, no. 4, pp. 1482-1498, 1962.

[14] J.-F. Lu, D.-S. Jeng, and W.-D. Nie, "Dynamic response of a pile embedded in a porous medium subjected to plane SH waves," Computers and Geotechnics, vol. 33, no. 8, pp. 404-418, 2006.

[15] J.-F. Lu and H.-Y. Yuan, "The sequence Fourier transform method for the analysis of a periodic viaduct subjected to nonuniform seismic waves," Acta Mechanica, vol. 224, no. 9, pp. 2143-2168, 2013.

[16] G. Bonnet, "Basic singular solutions for a poroelastic medium in the dynamic range," Journal of the Acoustical Society of America, vol. 82, no. 5, pp. 1758-1762, 1987.

[17] B. Xu, Dynamic Responses of the Saturated Poro-Elastic HalfSpace Due to a Moving Loads and Analysis of Vibration Isolation Effectiveness with Pile Rows, Shanghai Jiao Tong University, 2009.

[18] J. Wen, D. Yu, G. Wang, H. Zhao, and Y. Liu, "Elastic wave band gaps in flexural vibrations of straight beams," Chinese Journal of Mechanical Engineering, vol. 41, no. 4, pp. 1-6, 2005.

[19] C. Kittel, Introduction to Solid State Physics, Wiley, New York, NY, USA, 1996. 


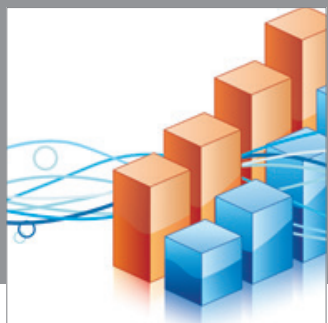

Advances in

Operations Research

mansans

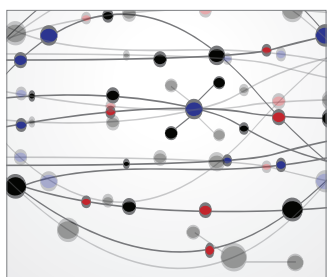

The Scientific World Journal
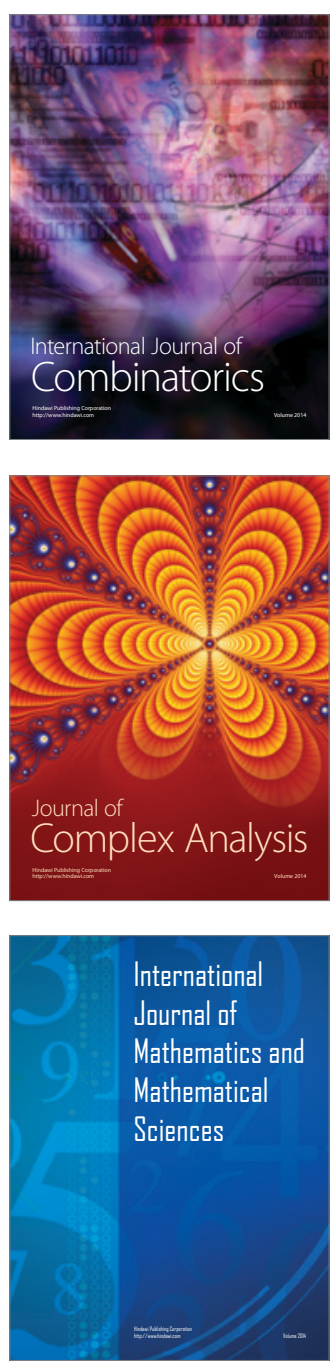
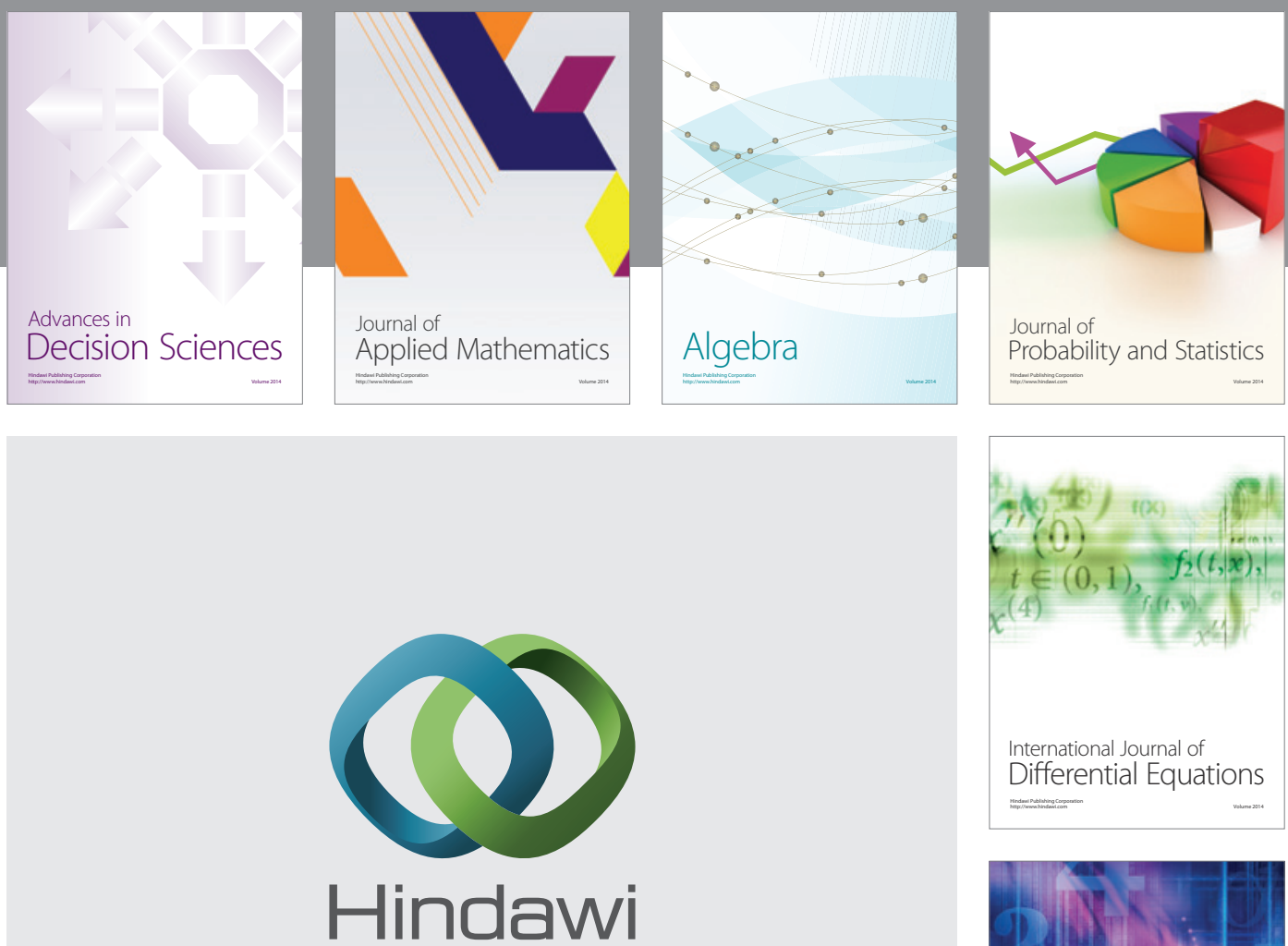

Submit your manuscripts at http://www.hindawi.com
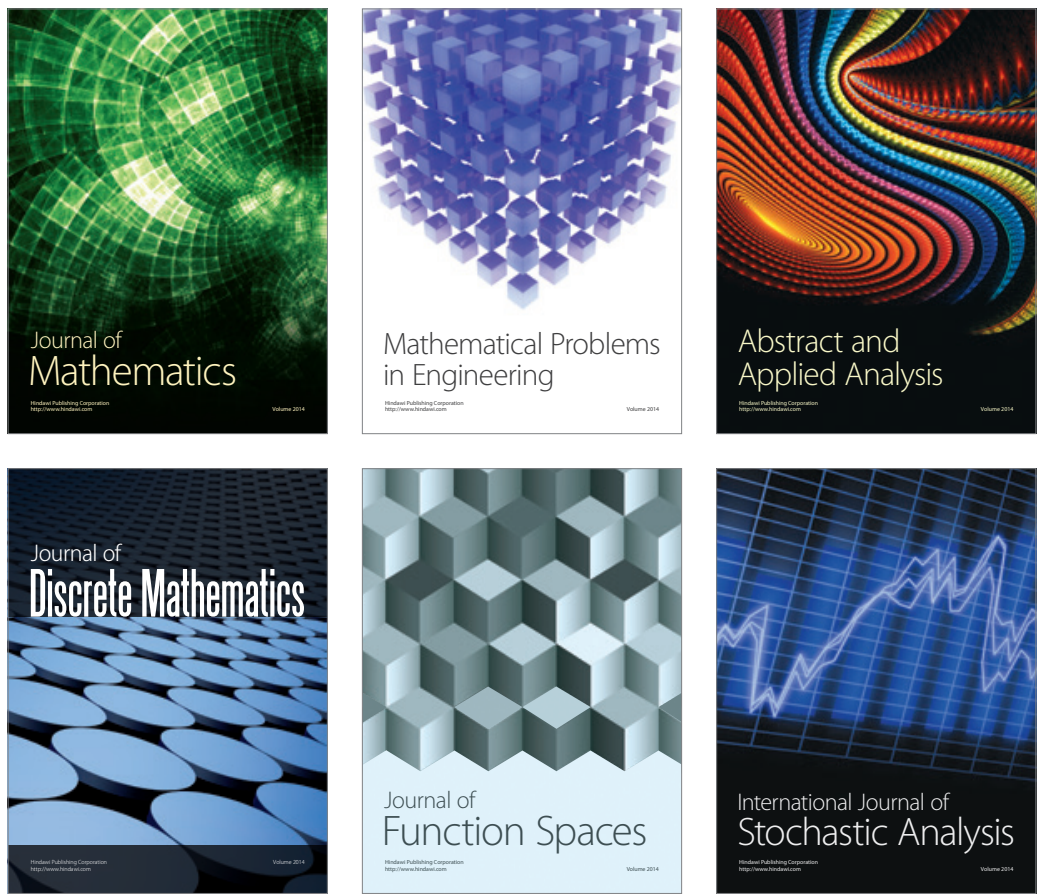

Journal of

Function Spaces

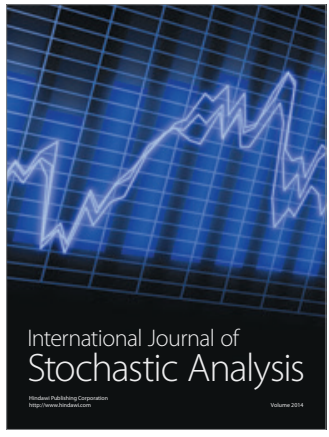

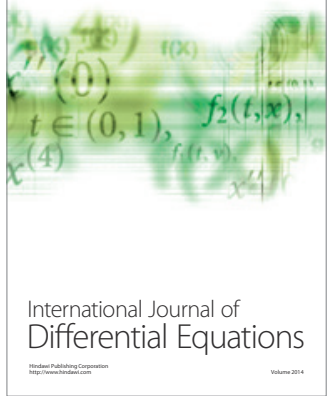
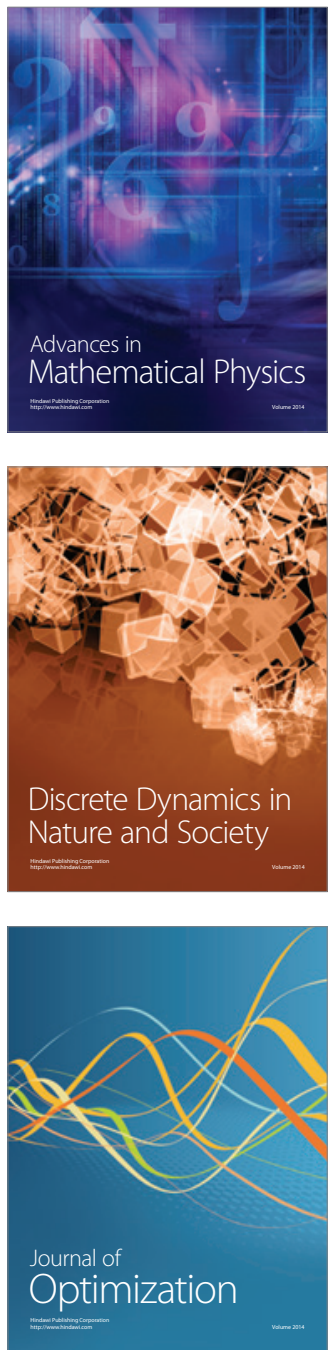